\title{
Prevalence of Undiagnosed Diabetes Mellitus amongst Hypertensive Patients Attending an Outpatient Clinic in Harare, Zimbabwe
}

\author{
Article by Pasipanodya Ian Machingura, Rosinah Murambiwah \\ Ph.D. in Public Health, Texila American University, Zimbabwe \\ E-mail: imachingura@yahoo.co.uk
}

\begin{abstract}
Diabetes mellitus is amongst the largest health emergencies of the $21^{\text {st }}$ century. However studies have reported that a substantial proportion of people with diabetes mellitus had not previously been diagnosed. On the other hand hypertension prevalence has been reported to be on the increase. Hypertension has also been associated with the development of diabetes mellitus. The study sought to determine the prevalence of undiagnosed diabetes mellitus in hypertensive patients attending an outpatient clinic in Harare, Zimbabwe.

A cross sectional study was conducted at Parirenyatwa Group of Hospitals Outpatient Clinic. All hypertensive patients attending the outpatient during the study period were given the information about the study. All hypertensive patients who were previously not diagnosed of diabetes mellitus who consented to participate in the study were consecutively enrolled. A questionnaire was administered and blood samples were collected for Glycosylated hemoglobin analysis. Glycosylated hemoglobin was analysed using a Mindray BS800 Chemistry Analyser.

One hundred and four hypertensive patients were enrolled into the study whose mean age was 60.91( \pm standard deviation of 14.06) years. Patients with duration of hypertension greater than 10 years constituted 34\% (35) of the study population. The prevalence of undiagnosed diabetes mellitus was $22.1 \%$ (23). Among the previously undiagnosed diabetic patients $56.5 \%$ (13) were females and $43.5 \%$ (10) males.

The prevalence of undiagnosed diabetes mellitus was found to be 22, 1\% among hypertensive patients which is higher than that reported in Kenya but lower than that reported in Uganda. Diabetes mellitus can be highly prevalent in hypertensive patients thus there is need for constant monitoring for the development of diabetes mellitus and further research using a national representative sample.
\end{abstract}

Keywords: Diabetes mellitus, pre-diabetes, glycosylated haemoglobin

\section{Introduction}

Diabetes mellitus is the amongst the largest health emergencies of the $21^{\text {st }}$ century causing life changing complications. Africa is reported to have the largest proportion of undiagnosed diabetes mellitus, it is estimated over two thirds of the people with diabetes mellitus are unaware of it(1). Diabetes mellitus and hypertension coexist in patients. The prevalence of hypertension is reported to be 1.5 to 2.0 times more in diabetes mellitus patients compared to non-diabetics whilst approximately a third of hypertension patients would develop diabetes mellitus. The coexistence of the two results in an increased risk and can accelerate vascular complications (2).

There is a complex cause effect relationship between hypertension and diabetes mellitus which is hypothesised. The new onset diabetes mellitus in hypertensive patients could be caused by treatment with antihypertensive drugs such as diuretics and beta blockers (3). Both diabetes mellitus and hypertension are manageable health conditions which can be controlled through use of medicines, exercise and diet. The detection of progenitors namely pre-diabetes and prehypertension by surveillance enables the early intervention and result in delay in the disease progression (2). The World Health Organisation does recommend cardiovascular risk 
Texila International Journal of Public Health

Volume 4, Issue 4, Dec 2016

assessment which does include glucose testing amongst patients presenting with hypertension (4). A third of all people with diabetes mellitus are reported that they may be undiagnosed and above $60 \%$ of the newly diagnosed diabetes mellitus patients are unaware of their condition until they develop complications (5).

There is paucity of documented data on prevalence of undiagnosed diabetes mellitus in high risk groups such as hypertensive patients in a clinical setting in Zimbabwe. Thus the study sought to determine the prevalence of undiagnosed diabetes mellitus in hypertensive patients attending an outpatient clinic in Harare, Zimbabwe.

\section{Methods}

\section{Ethical considerations}

The study was approved by the Joint Research Ethics Committee of the Parirenyatwa Group of Hospitals and University of Zimbabwe College of Health Sciences (JREC 342/15) and informed consent was obtained for all the study participants included in the study.

\section{Study design and study sites}

A cross-sectional study was carried out on hypertensive patients attending Parirenyatwa Group of Hospitals Outpatient Clinic, Harare, Zimbabwe in the period 08 February 2016 to 21 March 2016.ParirenyatwaGroup of Hospitals is at tertiary teaching hospital located in the capital city of Zimbabwe Harare.

\section{Study subjects}

All adult hypertensive patients (18 years and above) attending Parirenyatwa Group of Hospitals Outpatient Clinic were given information on study. All hypertensive patients who were not diagnosed of diabetes mellitus before who consented to participate in the study were consecutively enrolled.

\section{Data collection}

A questionnaire was administered on all adult hypertensive patients who consented to participate in study. A blood sample was collected into an ethylenediaminetetra acetic acid (EDTA) tube for glycosylated haemoglobin analysis(HbA1c) using vacuitaner method of blood collection.

\section{Sample analysis}

The samples collected in EDTA tubes were stored at 2 to 8 degrees Celsius and analysed for HbA1c within a week. The samples were thawed to room temperature and glycosyslated haemoglobin analysis was carried outon a Mindray BS 800 Chemistry Analyser(Mindray, Shenzhen, China). The assay kits, calibrators and standards were supplied by the manufacturer. The analyser was calibrated and then controls were assayed before the samples were analysed following manufacturers' recommendations.

\section{Data analysis}

Statistical Package for the Social Sciences version 8 data analysis package was used to analyse the data. Normally distributed data was analysed using mean and standard deviation. Normally distributed data was compared using the student $t$ test.

\section{Definitions}

The following case definitions were used:

- $\quad$ Non-diabetes, HbA1c < $6.0 \%$ (6)(7)

- $\quad$ Pre-diabetes, HbA1c between $6.0 \%$ and $6.4 \%(6)(7)$

- $\quad$ Diabetes mellitus, HbA1c $\geq 6.5 \%$ (6)(7) 


\section{Results}

One hundred and four hypertensive patients were recruited into the study of whom $64.4 \%$ (67) were female. The mean age of study participants was 60.91( \pm standard deviation of 14.06) years. $76.0 \%$ (79) of participants were on enalapril and $24.0 \%$ (25) were on atenolol antihypertensive drugs. The mean $\mathrm{HbA1c}$ was $5.8 \%$. There was no significant difference in mean HbAIc levels of females $(5.7 \%)$ and male $(5.9 \%)$ participants, (p value $=0.335$ ).

Patients with the duration of hypertension greater than 10 years constituted 34\% (35) of the study population and $81 \%$ (84) of participants had no family history of diabetes mellitus. Eighty six percent (89) of the study participants reported that they do not undertake regular exercise.

Table 1: To show the classification of study participants according to diabetes mellitus status

\begin{tabular}{|l|l|l|}
\hline Category & $\begin{array}{l}\text { Number of } \\
\text { patients }\end{array}$ & Percentage (\%) \\
\hline Non-Diabetes & 54 & 51.9 \\
\hline Pre-diabetes & 27 & 26.0 \\
\hline Diabetes mellitus & 23 & 22.1 \\
\hline Total & 104 & 100 \\
\hline
\end{tabular}

Table 1 shows the prevalence of undiagnosed diabetes mellitus was 22.1\% (23). Among diabetic hypertensive participants were $56.5 \%$ (13) females and $43.5 \%$ (10) males. Prediabetes constituted $26 \%$ (27) of participants with $66.7 \%$ (18) being females and $33.3 \%$ (9) were males. $51.9 \%$ (54) of study participants were non-diabetic and of these $66.7 \%$ (36) were females while $33.3 \%$ (18) were males.

\section{Discussion}

The coexistence of hypertension and diabetes mellitus does increase the risk of end organ damage, the incidences of cardiovascular disease and mortality. Whilst pre-diabetes is an independent risk factor for incident cardiovascular disease and mortality(8). Pre-diabetes is an intermittent stage of overt diabetes where blood glucose is higher than normal value but not high enough to meet diagnostic criteria of diabetes mellitus. The majority of diabetes mellitus patients are reported to go through a pre-diabetes phase for several years in which an opportunity exist to identify them and commence timely prevention. Studies have reported that the use of HbA1c is a highly standardised convenient test which exhibits low intraindividual variation which can be obtained at any time requiring no patient preparation and a sample is relatively stable at room temperature after collection. A strong correlation exists between average plasma glucose and HbA1c (5) which we used in this current study.

In this current study the prevalence of pre-diabetes mellitus was $26.0 \%$ whilst that of diabetes mellitus was 22.1\%. A study at a district hospital in Kiambu Kenya [2014] reported prevalence of pre-diabetes of $18 \%$ and $14 \%$ newly diagnosed diabetes mellitus(5). In as study at Mulago hospital in Uganda [2005-2006] prevalence of pre-diabetes mellitus was 50\% and newly diagnosed diabetes mellitus was $24 \%$ (9).A study at a tertiary hospital in Imo state of Nigeria [2011] reported a prevalence of pre-diabetes of 33.1\% amongst adult Nigerians with essential hypertension (10).

In Germany amongst hypertensive patients they reported prevalence of pre-diabetes of $39 \%$ and diabetes mellitus of $12 \%$ (11). Lower prevalence of pre-diabetes and diabetes mellitus are expected in developed countries due to their better glucose testing programmes. Whilst on the contrary higher rates of pre-diabetes and diabetes in developing countries are due to lack of rigorous screening which leaves many patients undiagnosed (9). 
Texila International Journal of Public Health

Volume 4, Issue 4, Dec 2016

Above three quarters of the patients were on enalapril an angiotensin converting enzyme inhibitor which is amongst the second line antihypertensive agents in Zimbabwe whilst the rest were on atenolol a beta blocker which is amongst the first line antihypertensive agents in Zimbabwe (12). Beta blockers have been associated with new onset diabetes amongst hypertension patients (3).

Eighty nine percent of patients reported that they do not undertake regular exercise which however has been reported that if it is combined with dietary intervention decrease incidence of type 2 diabetes mellitus in high risk group(13).

We recommend regular screening of diabetes mellitus amongst the hypertensive patients to diagnose pre-diabetes mellitus and prevent the development of diabetes mellitus which is associated with morbidity and mortality.

\section{Conclusion}

The prevalence of undiagnosed diabetes mellitus was found to be 22, $1 \%$ among hypertensive patients which is higher than that reported in Kenya but lower than that reported in Uganda. Diabetes mellitus can be highly prevalent in hypertensive patients thus there is need for constant monitoring for the development of diabetes mellitus and further research using a national representative sample.

\section{References}

[1] Bruno RM, Taddei S. New-onset diabetes in hypertensive patients and mortality: timing is everything. Eur Heart J. 2016 Mar 21;37(12):975-7.

[2] International Diabetes Federation. International Diabetes Federation Diabetes Atlas Seventh Edition [Internet]. 2015 [cited 2016 Jul 20]. Available from: www.diabetesatlas.org

[3] Iloh GUP. Risk Factors of Pre-Diabetes among Adult Nigerians with Essential Hypertension in a Resource-Constrained Setting of a Primary Care Clinic in Eastern Nigeria. Am J Health Res. 2013;1(3):56.

[4] Joshi SR, Saboo B, Vadivale M, Dani SI, Mithal A, Kaul U, et al. Prevalence of Diagnosed and Undiagnosed Diabetes and Hypertension in India—Results from the Screening India's Twin Epidemic (SITE) Study. Diabetes Technol Ther. 2012 Jan;14(1):8-15.

[5] Korhonen P, Aarnio P, Saaresranta T, Jaatinen P, Kantola I. Glucose Homeostasis in Hypertensive Subjects. Hypertension. 2008 Apr 1;51(4):945-9.

[6] Lüders S, Hammersen F, Kulschewski A, Venneklaas U, Züchner C, Gansz A, et al. Diagnosis of impaired glucose tolerance in hypertensive patients in daily clinical practice. Int $\mathrm{J}$ Clin Pract. 2005;59(6):632-8.

[7] Ministry of Health and Child Welfare Republic of Zimbabwe. 6th Essential Medicines list and standard treatment guidelines for Zimbabwe. 2011.

[8] Mutebi E, Nakwagala FN, Nambuya A, Otim M. Undiagnosed diabetes mellitus and impaired glucose tolerance among hypertensive patients in Mulago Hospital, Kampala, Uganda. Afr J Diabetes Med [Internet]. 2012 [cited 2016 Oct 28];20. Available from: http://www.africanjournalofdiabetesmedicine.com/articles/May_2012/AJDM\%20MAY\%20PP\%202023.pdf

[9] Meme N, Amwayi S, Nganga Z, Buregyeya E. Prevalence of undiagnosed diabetes and prediabetes among hypertensive patients attending Kiambu district Hospital, Kenya: a cross-sectional study. Pan Afr Med J [Internet]. 2015 [cited 2016 Oct 28];22. Available from: http://www.panafricanmed-journal.com/content/article/22/286/full/

[10] Organisation mondiale de la santé. Global status report on noncommunicable diseases 2014: attaining the nine global noncommunicable diseases targets; a shared responsibility. Geneva: World Health Organization; 2014.

[11] Organization WH, others. Use of glycated haemoglobin (HbA1c) in diagnosis of diabetes mellitus: abbreviated report of a WHO consultation. 2011 [cited 2016 Oct 28]; Available from: http://apps.who.int/iris/handle/10665/70523 
[12] Orozco LJ, Buchleitner AM, Gimenez-Perez G, Roqué i Figuls M, Richter B, Mauricio D. Exercise or exercise and diet for preventing type 2 diabetes mellitus. In: The Cochrane Collaboration, editor. Cochrane Database of Systematic Reviews [Internet]. Chichester, UK: John Wiley \& Sons, Ltd; 2008 [cited 2016 Oct 28]. Available from: http://doi.wiley.com/10.1002/14651858.CD003054.pub3

[13] The International Expert Committee. International Expert Committee Report on the Role of the A1C Assay in the Diagnosis of Diabetes. Diabetes Care. 2009 Jul 1;32(7):1327-34. 